\title{
Renormalization Group in Curved Space and the Problem of Conformal Anomaly
}

\author{
M. Asorey, \\ Departamento de Física Teorica, Universidad de Zaragoza, 50009, Zaragoza, Spain \\ E. V. Gorbar,* \\ Department of Applied Mathematics, University of Western Ontario, London, Ontario N6A 5B7, Canada \\ and I. L. Shapiro ${ }^{\dagger}$ \\ Departamento de Física, Universidade Federal de Juiz de Fora, Juiz de Fora, CEP: 36036-330, MG, Brazil \\ (Received on 11 October, 2005)

\begin{abstract}
Renormalization Group (RG) is a powerful method for investigating quantum effects of matter fields on curved background. The formalism of RG in curved space is well known since 1984, but its applications to cosmology and black hole physics require more knowledge and opens a new interesting field of study. We review recent results about the derivation of renormalization group in a mass-dependent scheme and also consider ambiguities of conformal anomaly using dimensional and covariant Pauli-Villars regularizations.
\end{abstract}

The Effective Action of vacuum (EA) is a most useful object to parametrize the quantum effects within quantum field theory in curved space-time $[1,2]$. Sometimes this approach is refereed to as semiclassical approximation, in order to emphasize that the metric is not quantized. The EA is a metricdependent functional, at one-loop level it consists of the classical action of vacuum and the contributions of closed loops of free matter fields. At higher loops the fields interactions become relevant. However, the practical derivations of the EA are mainly restricted by the one-loop order and here we shall also work in the framework of this approximation. In $d=4$ even the contributions of free matter fields are very complicated to calculate, and this task has been successful only for some particular cases. The word "particular" corresponds to the choice of both the model for the quantum field and the one for the background classical metric. The most remarkable example is the case of massless conformal invariant fields on the cosmological (homogeneous and isotropic) metric background, where the EA can be derived exactly $[3,4]$. This result has been obtained through integration of conformal anomaly $[5,6]$, showing the importance of anomaly and the related renormalization group for the analysis of the EA.

The investigation of EA in more complicated situations, in particular for massive fields, requires a choice of the approximation and of the calculational scheme. In this respect the seminal role belongs to the Renormalization Group (RG), which enables one to reduce the information about complicated non-local quantum contributions to the relatively simple dependence on a single parameter $\mu$. The most simple and most formal version of RG, based on the Minimal Subtraction $\overline{\mathrm{MS}}$ renormalization scheme, has been successfully applied to the quantum field theory in curved space (see, e.g. [2] for the

* On leave from Bogolyubov Institute for Theoretical Physics, 03143 Kiev, Ukraine

${ }^{\dagger}$ Also at Tomsk State Pedagogical University, Russia review and introduction). The weak side of the $\overline{\mathrm{MS}}$-based RG is the problem of interpretation of $\mu$ and related difficulties with applying RG, e.g. in cosmology.

An alternative way is to use some more sophisticated scheme of renormalization, which would not be universal one as the $\overline{\mathrm{MS}}$-based RG is, but which should be designed especially for the given application. Unfortunately, there is no covariant renormalization scheme except the $\overline{\mathrm{MS}}$ one. Furthermore, the known renormalization schemes (e.g. the momentum subtraction scheme) of renormalization are not really appropriate for the use in the gravitational framework. Still the momentum subtraction scheme enables one to extract some relevant information, part of it will be reviewed below. The main purpose of the original papers on the subject was to investigate the phenomenon of decoupling of massive fields at low energies for the free fields of different spin [7], the theory with Spontaneous Symmetry Breaking [8], for one particular example of interacting theory [9] and also to clarify some long-standing problem concerning the conformal anomaly [10].

The main goal of the works [7-9] was to formulate the effective approach for quantum field theory in curved spacetime. The notion of decoupling is an important aspect of effective approach. At classical level decoupling means that a heavy field doesn't propagate at low energies. This can be easily seen observing the propagator of a massive particle at low energy

$$
\frac{1}{k^{2}+M^{2}} \approx \frac{1}{M^{2}}+O\left(\frac{k^{2}}{M^{4}}\right), \quad k^{2} \ll M^{2} .
$$

The decoupling theorem explains how the suppression of the effects of heavy particles occurs at quantum level. Let us start from the pedagogical example and consider QED in flat space. The 1-loop vacuum polarization is

$$
-\frac{e^{2} \theta_{\mu v}}{2 \pi^{2}} \int_{0}^{1} d x x(1-x) \ln \frac{m_{e}^{2}+p^{2} x(1-x)}{4 \pi \mu^{2}},
$$

where $\theta_{\mu v}=\left(p_{\mu} p_{v}-p^{2} g_{\mu v}\right)$ and $\mu$ is the parameter of dimensional regularization. In the $\overline{\mathrm{MS}}$ renormalization scheme the 
$\beta$-function $\beta^{\overline{\mathrm{MS}}}$ results from acting $\frac{e}{2} \mu \frac{d}{d \mu}$ on the form factor of $\theta_{\mu v}$

$$
\beta_{e}^{\overline{\mathrm{MS}}}=\frac{e^{3}}{12 \pi^{2}}
$$

This $\beta$-function can not tell us about the decoupling. In order to define physical $\beta$-function, let us apply the mass-dependent renormalization scheme. Subtracting the divergence at $p^{2}=$ $M^{2}$ and taking derivative $\frac{e}{2} M \frac{d}{d M}$ we arrive at

$$
\beta_{e}=\frac{e^{3}}{2 \pi^{2}} \int_{0}^{1} d x x(1-x) \frac{M^{2} x(1-x)}{m_{e}^{2}+M^{2} x(1-x)} .
$$

The UV limit $\left(M \gg m_{e}\right)$ gives $\quad \beta_{e}=\beta_{e}^{\overline{\mathrm{MS}}}$ and in the IR limit $\left(M \ll m_{e}\right)$ we meet a quadratic decoupling compared to the UV $\beta$-function (Appelquist \& Carazzone theorem [11])

$$
\beta_{e}=\frac{e^{3}}{60 \pi^{2}} \cdot \frac{M^{2}}{m_{e}^{2}}+O\left(\frac{M^{4}}{m^{4}}\right) .
$$

In order to derive decoupling theorem for gravity we have considered massive fields on the classical metric background [7]. As we have already mentioned above, there is no completely covariant technique compatible with the massdependent renormalization schemes. We have performed calculations within the linearized gravity on the flat background $g_{\mu v}=\eta_{\mu \nu}+h_{\mu v}$. The corrections to the graviton propagator come from the standard Feynman diagrams [7] or may be derived using the covariant heat-kernel solution in the second order in curvature approximation $[12,13]$.

The polarization operator must be compared to the tensor structure of the vacuum Lagrangian

$$
L=-\frac{1}{16 \pi G}(R+2 \Lambda)+a_{1} C^{2}+a_{2} E+a_{3} \square R+a_{4} R^{2} .
$$

Here $C^{2}=C_{\mu v \alpha \beta}^{2}$ is the square of the Weyl tensor and $E$ is the integrand of the Gauss-Bonnet topological invariant (Euler characteristic) $E=R_{\mu \nu \alpha \beta}^{2}-4 R_{\mu \nu}^{2}+R^{2}$.

In the case of massive scalar we meet, after performing all the integrations, the following EA:

$$
\begin{aligned}
\bar{\Gamma}_{s}^{(1)} & =\int \frac{d^{4} x \sqrt{g}}{2(4 \pi)^{2}}\left\{\frac{m^{4}}{2} \cdot\left(\frac{1}{\varepsilon}+\frac{3}{2}\right)+\tilde{\xi} m^{2} R\left(\frac{1}{\varepsilon}+1\right)\right. \\
& \left.+\frac{1}{2} C_{\mu v \alpha \beta}\left[\frac{1}{60 \varepsilon}+k_{W}\right] C^{\mu v \alpha \beta}+R\left[\frac{\tilde{\xi}^{2}}{2 \varepsilon}+k_{R}\right] R\right\} .
\end{aligned}
$$

where $\frac{1}{\varepsilon}=\frac{1}{2-w}+\ln \left(\frac{4 \pi \mu^{2}}{m^{2}}\right), \quad \tilde{\xi}=\xi-\frac{1}{6}$. The formfactors for the higher derivative terms are

$$
\begin{aligned}
k_{W} & =k_{W}(a)=\frac{8 A}{15 a^{4}}+\frac{2}{45 a^{2}}+\frac{1}{150} \\
k_{R} & =k_{R}(a)=A \tilde{\xi}^{2}+\left(\frac{1-3 A}{18}+\frac{2 A}{3 a^{2}}\right) \tilde{\xi} \\
& +\frac{A}{9 a^{4}}-\frac{A}{18 a^{2}}+\frac{A}{144}+\frac{1}{108 a^{2}}-\frac{7}{2160}
\end{aligned}
$$

where we used notations

$$
A=1+\frac{1}{a} \ln \left|\frac{2-a}{2+a}\right|, \quad a^{2}=\frac{4 \square}{4 m^{2}-\square} .
$$

Obviously, constant formfactors mean zero $\beta$-functions for $\Lambda$ and $G$. The situation for the higher derivative terms is quite different. For the Weyl term the $\beta$-function is

$$
\beta_{1}=-\frac{1}{(4 \pi)^{2}}\left(\frac{1}{18 a^{2}}-\frac{1}{180}-\frac{a^{2}-4}{6 a^{4}} A\right) .
$$

In the UV and IR limits we have

$$
\begin{array}{r}
\beta_{1}^{U V}=-\frac{1}{(4 \pi)^{2}} \frac{1}{120}+O\left(\frac{m^{2}}{p^{2}}\right), \\
\text { and } \beta_{1}^{I R}=-\frac{1}{1680(4 \pi)^{2}} \frac{p^{2}}{m^{2}}+O\left(\frac{p^{4}}{m^{4}}\right) .
\end{array}
$$

The last formula is nothing but the Appelquist \& Carazzone theorem for gravity. Here $p$ is the momentum of the linearized gravitational field $h_{\mu v}$ on flat background.

For the $R^{2}$ term the situation is very similar. The bulky expressions for the $\beta$-function can be found in [7], the comparison of the UV and IR limits manifests standard quadratic decoupling

$$
\begin{aligned}
& \beta_{2}^{U V}=-\frac{1}{2(4 \pi)^{2}} \tilde{\xi}^{2}+O\left(\frac{m^{2}}{p^{2}}\right) \\
& \beta_{2}^{I R}=-\frac{p^{2} / m^{2}}{12(4 \pi)^{2}}\left[\tilde{\xi}^{2}-\frac{\tilde{\xi}}{15}+\frac{1}{630}\right]+O\left(\frac{p^{4}}{m^{4}}\right) .
\end{aligned}
$$

Similar results for the decoupling take place for the massive fermion and massive scalar cases [7]. The difference with the contributions of massive scalar consists in the coefficients of the same $a$-dependent terms. One important consequence of this difference can be observed in the theory with broken supersymmetry. If we assume that all particles of the MSM are very light compared to the $s$-particles, we arrive at the plot of the $\beta_{2}$-function for the local $\int R^{2}$-term (see Fig. 1 ). At this plot the value $a=2$ corresponds to the UV limit and the value $a=0$ corresponds to the IR limit. One can see that the sign of this $\beta$-function is changing on the way from UV to IR. This change of sign leads to the transition from stable to unstable regimes [14] in the model of anomaly-induced inflation [15].

In the theory with Spontaneous Symmetry Breaking (SSB) the situation is essentially more complicated. In this case, even at the tree level one has an infinite number of non-local terms in both vacuum and induced actions of gravity, and all those non-local terms in the vacuum action get renormalized at the quantum level. However, the standard decoupling law for the higher derivative terms holds in this case too [8].

An expansion $g_{\mu v}=\eta_{\mu v}+h_{\mu v}$ works well for higher derivative terms, but not for $\Lambda$ and $G$. Why did we obtain $\beta_{\Lambda}=\beta_{1 / G} \equiv 0$ in the momentum-subtraction scheme? Let us remind that the same $\beta$-functions are non-zero in the $\overline{M S}$-renormalization scheme [2]. In fact, the running in the momentum-subtraction scheme means the presence of a 


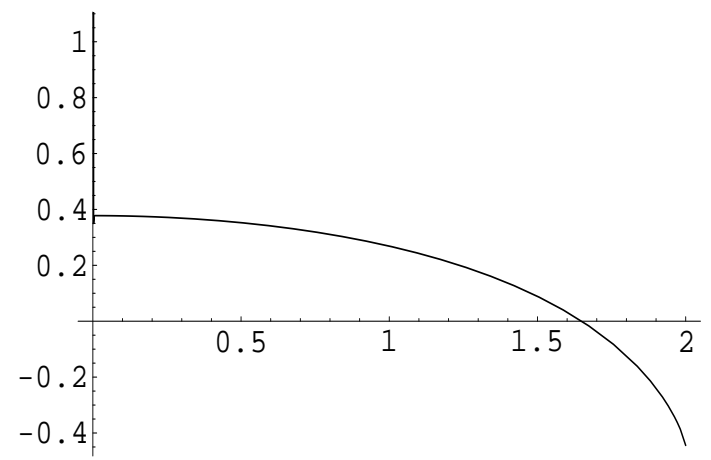

FIG. 1: The plot $\beta_{2}(a)$ demonstrating the change of sign between UV and IR.

$f(\square)=\ln \left(\square / \mu^{2}\right)$-like formfactor. In QED, in the UV limit we meet the term

$$
-\frac{e^{2}}{4} F_{\mu \nu} F^{\mu \nu}+\frac{e^{4}}{3(4 \pi)^{2}} F_{\mu \nu} \ln \left(-\frac{\square}{\mu^{2}}\right) F^{\mu \nu} .
$$

Similarly in gravity it is possible to insert

$$
C_{\mu v \alpha \beta} f(\square) C^{\mu v \alpha \beta} \quad \text { or } \quad R f(\square) R
$$

in the higher derivative sector. However, no insertion is possible for $\Lambda$ and $1 / G$, since $\square \Lambda=0$ and $\square R$ is a total derivative.

Does it mean that $\beta_{\Lambda}$ and $\beta_{1 / G}$ really equal zero? From our point of view the answer is negative, for otherwise we meet a divergence between the mass-dependent renormalization scheme and $\overline{M S}$-scheme in the UV where they are supposed to be the same. Perhaps calculations on a flat background are not appropriate for deriving the renormalization group equations for $\Lambda$ and $1 / G$. This hypothesis is quite natural, especially because flat space is not a classical solution in the presence of the cosmological constant. Let us notice that the practical derivation of the decoupling law for $\Lambda$ and $1 / G$ may have very interesting cosmological and astrophysical applications [16].

One of the applications of our expression for the effective action of massive fields (5) is the new way of analyzing the conformal anomaly - a typical phenomenon for the quantized massless fields on classical curved background [17].

As an example, consider scalar field

$$
L=\frac{1}{2}\left\{(\nabla \varphi)^{2}+m^{2} \varphi^{2}+\left(\tilde{\xi}+\frac{1}{6}\right) R \varphi^{2}\right\} .
$$

In the $m=0, \xi=1 / 6$ limit of (5) we obtain, in the $\int R^{2}$ sector,

$$
-\frac{1}{12 \cdot 180(4 \pi)^{2}} \int d^{4} x g^{1 / 2} R^{2} .
$$

Due to the identity

$$
-\frac{2}{\sqrt{-g}} g_{\mu v} \frac{\delta}{\delta g_{\mu \nu}} \int d^{4} x \sqrt{-g} R^{2}=12 \square R .
$$

the result (10) provides a perfect fit with the $\square R$ term in the local (means with respect to local conformal symmetry) conformal anomaly obtained by the point-splitting method [18], $\zeta$-regularization [19] and other methods (see [1] for the review)

$$
<T_{\mu}^{\mu}>=\frac{1}{360(4 \pi)^{2}}\left[3 C^{2}-E+2 \square R\right],
$$

where $C^{2}=C^{2}(4)=R_{\mu v \alpha \beta}^{2}-2 R_{\mu \nu}^{2}+(1 / 3) R^{2}$ is the square of the Weyl tensor at $n=4$ and $E=R_{\mu \nu \alpha \beta}^{2}-4 R_{\mu \nu}^{2}+R^{2}$ is the integrand of the Gauss-Bonnet invariant. We added these two terms for consistency, despite our main attention will be paid to the $\square R$ term. Let us notice that the above result has been obtained via the massless conformal limit in the massive and non-conformal (generally, $\xi \neq 1 / 6$ ) theory. Indeed, for any $\xi \neq 1 / 6$, the $\int R^{2}$ term is subject of infinite renormalization procedure and therefore the above limit is ambiguous. Indeed, this situation may look quite trivial, because the term of our interest is local. However, a relevant detail is that, in the conformal theory, the counterterm of $\int R^{2}$ type is not needed, hence one can thing that the ambiguity is nothing but a consequence of a "wrong" choice of regularization scheme.

The anomaly can be seen as a consequence of an infinite regularization of the higher derivative terms in the vacuum action. The simplest way to derive the conformal anomaly is using the dimensional regularization [6], however this calculation meets certain problem. The usual scheme of calculation [6] is as follows. The renormalized 1-loop EA is a sum of three parts

$$
\Gamma_{\text {ren }}^{(1)}=S_{v a c}+\bar{\Gamma}^{(1)}+\Delta S_{v a c}
$$

where $S_{v a c}$ is the classical vacuum action. Indeed, this action can be chosen to be conformal invariant for the case of conformal matter [20]. Furthermore, $\bar{\Gamma}^{(1)}$ is the non-renormalized quantum correction, which is divergent, conformal invariant (as far as the matter field was formulated conformal invariant in $n \neq 4$ and non-local. Finally,

$$
\Delta S_{v a c}=-\frac{1}{(4 \pi)^{2}(n-4)}\left(3 C^{2}-E+2 \square R\right)
$$

is the local counterterm which is not conformal invariant is $n \neq 4$. Obviously, the anomaly comes from the unique nonconformal component, that is $\Delta S_{v a c}$.

$$
T=<T_{\mu}^{\mu}>=-\frac{2}{\sqrt{g}} g_{\mu v} \frac{\delta \Delta S_{v a c}}{\delta g_{\mu v}} .
$$

For the $C^{2}$ and $E$ terms the application of this formula leads us, unambiguously, to the expression (12). Let us now consider the remaining term and see that in this case the situation is more complicated. Indeed

$$
\frac{\delta}{\delta g_{\mu \nu}} \int d^{n} x \sqrt{g}(\square R) \equiv 0 .
$$

This shows that, in the dimensional regularization, the $\int \square R$ type counterterm does not contribute to the $\square R$ term in the 
anomaly. Of course, this is not a natural output, especially because the anomaly for the global conformal symmetry does depend on all counterterms. Furthermore, other regularization schemes also link the two coefficients in a direct way, so here we meet a divergence between the results obtained in distinct regularizations.

According to [6], the $\square R$ term in the anomaly is coming from the $\int C^{2}$-type counterterm via the identity

$$
\left.2 g_{\mu \nu} \frac{\delta}{\delta g_{\mu \nu}} \int d^{n} x \sqrt{g} \frac{C^{2}(4)}{n-4}\right|_{n \rightarrow 4}=\left(C^{2}-\frac{2}{3} \square R\right) .
$$

Hence $T=-(2 / 3) \beta_{W e y l} \cdot \square R+\ldots$. For the scalar and spinor fields this relation gives the same result as other regularization schemes. However, for the vector field and also for the higher derivative scalar [3] and fermion [22] the coefficients are different. Hence it is reasonable to look again at the definition (13).

Why we have to choose $\Delta S_{v a c}=\int \sqrt{g} C^{2}(4)$ ?. In fact, if we choose the square of the Weyl tensor in an arbitrary dimension $d$,

$$
C^{2}(d)=R_{\mu v \alpha \beta}^{2}-\frac{4}{d-2} R_{\mu v}^{2}+\frac{2}{(d-1)(d-2)} R^{2},
$$

where $d=4+O(n-4)$, the corresponding counterterm will be perfectly consistent with its destination, for it is local and cancels the divergence in the one-loop EA. However the result for the $(\square R)$ term in the anomaly essentially depends on the choice of $d$. E.g. for $C^{2}(n)$ there is no $\square R$ term at all and for the $d=n+\gamma \cdot[n-4]$ the $\square R$ term is proportional to an arbitrary parameter $\gamma$. We can conclude that the dimensional regularization does not predict the coefficient of the $\square R$ term in the anomaly. Let us notice that the change of parameter $\gamma$ in the counterterm which we add to $\bar{\Gamma}^{(1)}$ by hands is completely equivalent to adding a finite $\int R^{2}$ term to the classical action. Adding this term is absolutely safe procedure, because it is not a subject of infinite regularization. From the point of view of renormalizability this term is not necessary but it is not forbidden either. Hence the arbitrariness we have found in the dimensional regularization is nothing else but the consequence of the identity (11). This arbitrariness takes place also for other fields and finally the coefficient $\alpha^{\prime}$ in the general expression for the anomaly

$$
<T_{\mu}^{\mu}>=\left[w C^{2}+b E+\alpha^{\prime} \square R\right]
$$

can not be defined within the dimensional regularization. We remark that this arbitrariness is exactly the same we already met when taking the massless conformal limit in the effective action of a massive scalar theory with an arbitrary $\xi$.

In order to complete the story we can answer the following question: is it possible to observe an arbitrariness in $\alpha^{\prime}$ in some other regularization? In order to address this issue we need another example of covariant regularization which should admit certain freedom in choosing regularization parameters. And our result for the effective action (5) enables us to build the regularization of this sort.

Consider covariant version of the Pauli-Villars regularization, known from Yang-Mills and Chern-Simons theories
$[23,24]$. The Pauli-Villars regularization implies introducing massive auxiliary fields with distinct Grassmann parity which cancel all (quartic, quadratic, log.) divergences in a covariant way

$$
S_{\mathrm{reg}}=\sum_{i=0}^{N} \int d^{4} x \sqrt{g}\left\{\left(\nabla \varphi_{i}\right)^{2}+\left(\xi_{i} R+m_{i}^{2}\right) \varphi_{i}^{2}\right\} .
$$

The physical scalar field $\varphi \equiv \varphi_{0}$ is conformal $\xi=1 / 6, m_{0}=0$ and bosonic $s_{0}=1$.

On the other hand, the Pauli-Villars regulators $\varphi_{i} \quad(i=$ $1, \ldots, N)$ are massive $m_{i}=\mu_{i} M \neq 0$ and can have either bosonic $s_{i}=1$ or fermionic statistics $s_{i}=-2$. Since they are not observed, there is no violation of the spin-statistics theorem here.

After UV limit $M \rightarrow \infty$ we arrive at the vacuum EA. The calculation is based on our result (5), as we already mentioned. We assume that the Pauli-Villars regulators might have non-conformal couplings $\xi_{i} \neq \frac{1}{6}$. The regularized effective action is

$$
\bar{\Gamma}_{\text {reg }}^{(1)}=\lim _{\Lambda \rightarrow \infty} \sum_{i=0}^{N} s_{i} \bar{\Gamma}_{\mathrm{i}}^{(1)}\left(m_{i}, \xi_{i}, \Lambda\right)
$$

where $\Lambda$ is an auxiliary momentum cut-off. All divergences cancel out due to the Pauli-Villars conditions, which have, in our case, the following form:

$$
\begin{gathered}
\sum_{i=1}^{N} s_{i}=-1 ; \quad \text { (quartic divs.) } \\
\sum_{i=1}^{N} s_{i} \mu_{i}^{2}=\sum_{i=1}^{N} s_{i}\left(\xi_{i}-\frac{1}{6}\right)=0 ; \quad \text { (quadratic) } \\
\sum_{i=1}^{N} s_{i} \mu_{i}^{4}=\sum_{i=1}^{N} s_{i}\left(\xi_{i}-\frac{1}{6}\right)^{2}=0 ; \quad \text { (log.) }
\end{gathered}
$$

A simple solution of these equations is

$$
\begin{gathered}
s_{1,2,3,4,5}=(1,4,-2,2,-2), \\
\mu_{1,2,3,4,5}^{2}=(4,3,1,3,4), \quad \xi_{i}=\mu_{i}^{2}+1 / 6 .
\end{gathered}
$$

Alternatively we can choose all $\xi_{i}=1 / 6$, so there is an arbitrariness here.

The conformal anomaly in the covariant Pauli-Villars regularization is given by

$$
T=\frac{1}{(4 \pi)^{2}}\left[\frac{1}{180} E-\frac{1}{120} C^{2}+\left(12 \delta-\frac{1}{180}\right) \square R\right]
$$

where

$$
\delta=\sum_{i=1}^{N} s_{i}\left(\xi_{i}-\frac{1}{6}\right)^{2} \ln \mu_{i}^{2}
$$


Again, we meet an ambiguity. It is easy to see that, exactly as in the dimensional regularization case, the ambiguity is related to the freedom to add the finite $\int \sqrt{g} R^{2}$-term to the classical vacuum action.

The only way to fix the ambiguity in $\alpha^{\prime}$ is through a special renormalization condition for the $\int \sqrt{g} R^{2}$-term. In the free scalar theory this looks as an additional complication, because this term is not renormalized. But as we know from the literature [21], in the interacting theory the $\int \sqrt{g} R^{2}$-type counterterm emerges anyway at higher loops and, therefore, this extra condition does not lead to an essential modification of the theory structure. At that point one has to remember that the dynamical effect comes from the $\int \sqrt{g} R^{2}$-term, and not from the total derivative $\int \sqrt{g} \square R$. Looking from this perspective, we can see that the renormalization condition for the $\int \sqrt{g} R^{2}$-term is fixing the initial point of the renormalization group trajectory for the corresponding coupling, and not the shape of the trajectory. In other words, the curve shown at the Figure 1 remains valid if we impose the renormalization condition for the $\int \sqrt{g} R^{2}$-term in a proper way. As a result the model of anomaly-induced inflation is not jeopardized by the $\alpha^{\prime}$ ambiguity in the conformal anomaly.

In conclusion, we have established the background of an effective approach to QFT in curved space-time. In particular, the first derivation of the renormalization group in a physical mass-dependent scheme has been performed. Unfortunately, the calculation was possible only in the framework of a linearized gravity approximation, that is why we did not obtain the physical $\beta$-functions for the cosmological and Newton constants. The derivation of EA on a more complicated backgrounds in necessary for obtaining these $\beta$-functions, which can help us in order to prove or disprove the possibility of a time-dependent cosmological constant [16]. The same calculation is vital for further theoretical development of the anomaly-induced inflation model [15]. An important application of our calculation [7] of the effective action for massive scalar field is better understanding of the ambiguity in the conformal anomaly in $n=4$, the issue which produced a long-standing doubts [17]. We have seen that the dimensional regularization does not enable one to predict a coefficient $\alpha^{\prime}$ and that a similar ambiguity takes place in other calculational schemes, including taking a conformal limit in a massive theory and in the powerful Pauli-Villars regularization. At the same time the ambiguity concerns only $\int \sqrt{g} \square R$ in the anomaly and the induced dynamics coming from the vacuum effective action can be made completely unambiguous by introducing the $\int \sqrt{g} R^{2}$-term into classical action and imposing the corresponding renormalization condition at quantum level.

\section{Acknowledgments}

The work of M.A. is partially supported by CICYT (grant FPA2004-02948) and DGIID-DGA (grant2005-E24/2). The work of E.V.G. was supported by the Natural Sciences and Engineering Research Council of Canada. (I.Sh.) is grateful to CNPq and FAPEMIG and ICTP for permanent support and also to ICTP, Theory Group at CERN and to Departamento de Física Teórica, Universidad de Zaragoza for kind hospitality.
[1] N.D. Birell and P.C.W. Davies, Quantum Fields in Curved Space (Cambridge Univ. Press, Cambridge, 1982).

[2] I.L. Buchbinder, S.D. Odintsov and I.L. Shapiro, Effective Action in Quantum Gravity (IOP, Bristol, 1992).

[3] R.J. Reigert, Phys. Lett. B 134, 56 (1980); E.S. Fradkin and A.A. Tseytlin, Phys. Lett. B 134, 187 (1980).

[4] I.L. Shapiro and A.G. Jacksenaev, Phys. Lett. B 324, 286 (1994).

[5] S. Deser, M.J. Duff, and C. Isham, Nucl. Phys. B 111, 45 (1976).

[6] M.J. Duff, Nucl. Phys. B 125, 334 (1977).

[7] E.V. Gorbar, I.L. Shapiro, JHEP 02 (2003) 021; JHEP 06, 004 (2003).

[8] E.V. Gorbar, I.L. Shapiro, JHEP 02, 060 (2004).

[9] G. de Berredo-Peixoto, E.V. Gorbar, and I.L. Shapiro, Class. Quant. Grav., 21, 2281 (2004); [hep-th/0311229].

[10] M. Asorey, E.V. Gorbar, and I.L. Shapiro, Class. Quant. Grav. 21, 163 (2003)

[11] T. Appelquist, J. Carazzone, Phys. Rev. D 11, 2856 (1975).

[12] I. G. Avramidi, Yad. Fiz. (Sov. Journ. Nucl. Phys.) 49, 1185 (1989).

[13] A.O. Barvinsky, G.A. Vilkovisky, Nucl. Phys. B 333, 471 (1990).

[14] I.L. Shapiro, Int. Journal of Mod. Phys. D 11, 1159 (2002); [Hep-ph/0103128].

[15] J.C. Fabris, A.M. Pelinson, and I.L. Shapiro, Nucl. Phys. B 597, 539 (2001);
I.L. Shapiro, J. Solà. Phys. Lett. B 530, 10 (2002).

A.M. Pelinson, I.L. Shapiro, and F.I. Takakura, Nucl. Phys. B 648, 417 (2003).

[16] I. Shapiro, J. Solà, Phys. Lett. 475, 236 (2000); JHEP 0202, 006 (2002);

I.L. Shapiro, J. Solà, C. España-Bonet, and P. Ruiz-Lapuente, Phys. Lett. B 574, 149 (2003);

I.L. Shapiro, J. Solà, and H. Stefancic, JCAP 0501, 012 (2005).

[17] M.J. Duff, Class. Quant. Grav. 11, 1387 (1994).

[18] S.M. Christensen, Phys. Rev. D 14, 2490 (1976); 17D, 948 (1978); 14D, 2490 (1976).

[19] S.W. Hawking, Comm. Math. Phys. 55, 133 (1977).

[20] This statement must be taken with caution in the case of interacting fields [21].

[21] S.J. Hathrell, Ann. Phys. 139; 142 (1982).

[22] G. de Berredo-Peixoto and I.L. Shapiro, Phys. Lett. B 514, 377 (2001); [hep-th/0101158].

[23] A.A. Slavnov, Theor. Math. Phys. 33, 210 (1977). T.D. Bakeyev and A.A. Slavnov, Mod. Phys. Lett. A 11, 1539 (1996).

[24] M. Asorey and F. Falceto, Nucl. Phys. B 327, 427 (1989); Phys. Rev. D 54, 5290 (1996).

M. Asorey, F. Falceto, J. L. López, and G. Luzón, Nucl. Phys. B 429, 344 (1994).

J.L. López, Universalidad y Regularización Ultravioleta en Teorías Gauge (Ph.D. dissertation, Zaragoza University, 1995). 